Sílvia Helena de Carvalho Sales-Peres 1 José Roberto de Magalhães Bastos 2

\section{Perfil epidemiológico de cárie dentária em crianças de 12 anos de idade, residentes em cidades fluoretadas e não fluoretadas, na Região Centro-Oeste do Estado de São Paulo, Brasil}

\author{
An epidemiological profile of dental caries \\ in 12-year-old children residing in cities with \\ and without fluoridated water supply in the central \\ western area of the State of São Paulo, Brazil
}

1 Universidade Paulista. Rua Luiz Leborato $s / n$, Quarteirão 2, Bauru, SP 17048-290, Brasil.

shcperes@usp.br

2 Departamento de Odontopediatria, Ortodontia e Saúde Coletiva, Faculdade de Odontologia de Bauru, Universidade de São Paulo. Al. Otávio Pinheiro Brisola 9-75, Bauru, SP 17043-101, Brasil. jrbastos@fob.usp.br

\begin{abstract}
This study aimed to analyze the epidemiological profile of dental caries in the central western region of the State of São Paulo, Brazil, focusing on 12-year-old children by gender, comparing the DMFT index in fluoridated and non-fluoridated cities. The study used data from the Epidemiological Surveys in the State of São Paulo, 1998, pertaining to the 10th Regional Health Directorate, including 8 cities. The sample consisted of 485 schoolchildren, both boys and girls, distributed by city. In Pederneiras (a medium-sized city with a fluoridated water supply), DMFT was 7.06, higher than the Brazilian national mean for 1986. Boys showed a higher prevalence of dental caries than girls, showing a new trend for dental caries in the region. There was no statistically significant difference between DMFT in municipalities of the same size, regardless of the presence or absence of fluoride in the water supply, thus suggesting a "convergence" phenomenon, possibly due to the intake of other sources of fluoride and the presence of a "halo" effect. Prevalence of caries in the region was "high", with a DMFT of 4.82, thus failing to reach the goals set for the year 2000.
\end{abstract}

Key words Dental Caries; DMF Index; Fluorine

Resumo O objetivo deste estudo pautou em analisar o perfil epidemiológico de cárie dentária na Região Centro-Oeste do Estado de São Paulo, Brasil, em crianças de 12 anos de idade, segundo o gênero, comparando o índice CPOD em municípios fluoretados e não fluoretados. Foram utilizados os dados do Levantamento Epidemiológico do Estado de São Paulo - 1998, referentes à Direção Regional de Saúde - DIR X, representada por oito municípios. A amostra foi composta por 485 escolares, de ambos os gêneros, distribuídos pelos municípios. Os resultados demonstraram que na cidade de Pederneiras (médio porte, com flúor) o CPOD foi igual a 7,06, valor maior do que o encontrado no Brasil em 1986. A maior prevalência de cárie dentária ocorreu nos meninos, quando comparados às meninas, demonstrando uma nova tendência à cárie dentária na região centro-oeste. Não houve diferença estatisticamente significante entre CPOD de municípios de mesmo porte, independente da presença ou ausência de flúor na água, sugerindo o fenômeno da convergência. Possivelmente, pela ação da ingestão de outras fontes de flúor e a presença do efeito "halo". A prevalência de cárie na região esteve "alta", com CPOD igual a 4,82, não atingindo as metas para o ano 2000.

Palavras-chave Cárie Dentária; Índice CPO; Flúor 


\section{Introdução}

O grande problema da Odontologia não é apenas o conhecimento da prevalência e incidência da cárie dentária, mas, principalmente, a resolução dos problemas causados nos indivíduos e nas populações. Pinto (1997) afirma que o quadro epidemiológico brasileiro, em termos de saúde bucal, reflete com nitidez algumas das principais características da nossa sociedade: economia não estável, agudas desigualdades salariais e sociais, sistema educacional com ênfase na formação de nível superior, produção agrícola voltada para a exportação, grande número de pessoas de baixa renda e em estado de pobreza relativa ou absoluta. As tendências mundiais em relação à saúde bucal demonstram o declínio na incidência e prevalência de cárie (Murray, 1994) e o aumento da prevalência da fluorose (Angelillo et al., 1999); tais ocorrências não estão distribuídas de forma homogênea em toda a população. Há concentração da doença cárie e as necessidades de tratamento em uma pequena parcela da população (20-40\%), sendo que a maior parte apresenta-se praticamente livre de cárie (40-60\%), esta ocorrência é conhecida como "fenômeno da polarização". De Liefde (1998) avaliou o declínio da cárie na Nova Zelândia, durante os últimos quarenta anos. A queda da prevalência de cárie vem sendo observada desde os anos 50 , acompanhada por uma mudança no padrão bucal da doença. As regiões fluoretadas e não fluoretadas apresentaram o fenômeno da convergência, a partir dos anos 70 , com a introdução do uso de dentifrício fluoretado, complementado ainda pela suplementação de flúor, comidas e antibióticos fluoretados, o que parece ter corroborado para a mudança do perfil epidemiológico da cárie dentária.

No Brasil, foram realizados dois grandes Levantamentos Epidemiológicos Nacionais Sobre Cárie Dentária, sendo o primeiro em 1986 (MS, 1988), período em que o índice CPOD (dentes cariados, perdidos e/ou obturados) aos 12 anos de idade, foi igual a 6,67, e o segundo em 1996 (MS, 1996), observando-se o CPOD aos 12 anos igual a 3,12. Diferentes métodos preventivos foram aplicados nas populações com o objetivo de reduzir a prevalência de cárie dentária nessa idade, considerada pela Organização Mundial da Saúde (OMS) como a idade índice para comparações entre diferentes países e populações.

Narvai et al. (1999) investigaram o declínio na experiência de cárie em dentes permanentes de escolares brasileiros durante o final do século XX, e concluíram que a fluoretação das águas de abastecimento público, a adição de compostos fluoretados aos dentifrícios e a descentralização do sistema de saúde brasileiro são fatores que devem ser considerados para compreender o processo de declínio de cárie dentária. Pereira et al. (2000) estudaram a prevalência de cárie e fluorose em cidade brasileira não fluoretada, em Iracenópolis, através da análise das tendências de declínio e sua correlação aos dentifrícios fluoretados. Compararam estudos transversais em 1991, 1995 e 1997, onde verificaram o declínio de cárie e o aumento da fluorose. Estes resultados são concordantes com muitas pesquisas realizadas em municípios fluoretados e não fluoretados (Clark \& Berkowitz, 1997; Dini et al., 1998; Morgan et al., 1998) que apresentaram o fenômeno da convergência. O estudo de Silva \& Maltz (2001), realizado em Porto Alegre, com crianças aos 12 anos de idade nos anos de 1998/1999, correlacionou a prevalência de cárie e a fluorose em mil indivíduos. A média do CPOD foi de 2,22 e a prevalência de escolares com fluorose foi de 52,9\%. O grande número de lesões de cárie concentrou-se em poucos indivíduos. Somente $14,4 \%$ da amostra foi responsável por $51,2 \%$ da experiência de cárie, enquanto $48,8 \%$ do restante da experiência de cárie correspondeu a mais de $80 \%$ da amostra, o fenômeno da polarização. Ressaltaram também, a importância dessa informação para o planejamento em saúde pública.

O Levantamento Epidemiológico de Saúde Bucal do Estado de São Paulo de 1998 (FSP-USP, 1999), que foi realizado sob a coordenação da Faculdade de Saúde Pública da Universidade de São Paulo, abrangeu 24 Direções Regionais de Saúde (DIRs), representadas por 133 municípios e 89.114 participantes, distribuídos em faixas e grupos etários. Os resultados foram elaborados por DIR, e não por município, dando uma visão da região como um todo. A hipótese que norteou este trabalho foi de que há necessidade de uma análise mais acurada da complexidade da realidade, combinando conhecimentos clínicos e preventivos com informações gerais sobre os padrões de desenvolvimento social, econômico e político, identificando os grupos polarizados que mais necessitam de atenção odontológica, redirecionando os recursos e oferecendo soluções melhores ou mais oportunas para o extenso volume de problemas existentes. O objetivo deste estudo foi descrever as condições de saúde bucal das crianças residentes na DIR-X, situada na região centro-oeste do Estado, com 12 anos de idade. Foram analisadas segundo o gênero, o porcentual de crianças livres de cárie e as que necessitam de tratamento, as correlações entre cidades 
fluoretadas e não fluoretadas, e a comparação entre o padrão de saúde bucal na região com as metas da OMS para o ano 2000 (WHO, 1981).

\section{Material e método}

Este estudo tomou como base os dados da DIR$\mathrm{X}$, localizada na região centro-oeste do Estado de São Paulo, constituída por 41 cidades. No primeiro estágio, foram sorteadas as unidades amostrais primárias (municípios), no segundo, as unidades amostrais secundárias (escolas) e no terceiro, os elementos amostrais (indivíduos). Foram sorteados oito municípios da DIR-X para participar do levantamento, divididos segundo o porte (grande, médio e pequeno) e a presença ou ausência de flúor na água de abastecimento. As informações obtidas permitiram estratificar a amostra segundo gênero, grupo étnico, tipo de escola (pública ou particular) e localidade da escola (urbana ou rural). A população de referência para 12 anos de idade estava representada por 533 escolares, sendo compilados os dados de 485 formulários, os quais não apresentaram informações incorretas nas respectivas caselas. Alguns formulários apresentavam valores incorretos, portanto foram eliminados. A cidade que teve maior perda de dados foi Bauru, não aproveitando 21 formulários. Os municípios participantes foram: Cabrália Paulista e Paulistânia, de pequeno porte sem flúor na água; Boracéia e Pongaí, de pequeno porte com flúor na água; Barra Bonita, de médio porte sem flúor; Pederneiras, de médio porte com flúor; Jaú, de grande porte sem flúor e Bauru, de grande porte com flúor. As condições dentárias e necessidades de tratamento dos escolares foram registradas por município. A analise estatística foi descritiva, utilizando-se ANOVA a dois critérios para comparações do CPOD entre porte e fluoretação de águas. Quando esta indicou diferença estatisticamente significante, aplicou-se o teste de Tukey, para comparações múltiplas. Em todos os testes utilizados adotou-se nível de significância de $5 \%$.

\section{Resultados}

A análise do perfil epidemiológico de cárie dentária foi realizada por município, avaliando-se o índice CPOD aos 12 anos de idade, segundo o gênero. A maior prevalência ocorreu nos meninos, na grande maioria dos municípios, exceto em Pongaí, haja vista o tamanho reduzido da amostra. Os valores do CPOD en- contrados na região foram altos, e as cidades de médio porte apresentaram a maior concentração do problema (Tabela 1; Figura 1). A maioria dos escolares aos 12 anos não atingiram as metas da OMS para o ano 2000, sendo que só nos municípios de Bauru e Jaú, mais da metade dessas crianças atingiram esta meta (Figura 2).

O percentual das crianças livres de cárie em cada município (Figura 3), é muito heterogêneo, estando alguns municípios classificados, segundo a OMS, em baixa prevalência (mais de $20 \%$ de livres de cárie), Bauru e Jaú; moderada (5-20\%), Pederneiras, Boracéia, Pongaí e Barra Bonita e em alta (menos de 5\%), Cabrália Paulista e Paulistânia. Os municípios de médio e pequeno porte concentram o menor número de crianças livres de cárie, e cidades como Bauru e Jaú, o maior percentual. As necessidades de tratamento odontológico foram elevadas em toda a região, sendo que, no particular, os melhores dados são os de Jaú, com 59\% dos escolares não necessitando de tratamento odontológico (Figura 4). Não houve diferença estatisticamente significante entre o CPOD de municípios de mesmo porte, independente da presença ou ausência de flúor na água, sugerindo o fenômeno da convergência, conforme a Tabela 2, onde o teste de Tukey permite observar o fenômeno, de acordo com o porte do município (pequeno, médio, grande) e a presença ou ausência de flúor na água de abastecimento público (Tabela 2).

\section{Discussão}

O declínio da prevalência e severidade da cárie no Brasil e no mundo é fato reconhecido. Vários fatores colaboram para essa tendência, e é

Tabela 1

Índice CPOD aos 12 anos de idade, para cada município, segundo gênero.

\begin{tabular}{lcccc}
\hline Município & Masculino & CPOD & Feminino & CPOD \\
\hline Barra Bonita & 50 & 6,68 & 32 & 5,87 \\
Bauru & 37 & 3,08 & 38 & 2,76 \\
Boracéia & 18 & 3,33 & 14 & 2,35 \\
Cabrália Paulista & 21 & 5,19 & 18 & 4,55 \\
Jaú & 35 & 4,11 & 53 & 3,01 \\
Paulistânia & 32 & 5,15 & 26 & 4,30 \\
Pederneiras & 50 & 8,60 & 45 & 5,35 \\
Pongaí & 10 & 3,40 & 6 & 3,50 \\
Total & 253 & 5,52 & 232 & 4,05 \\
\hline
\end{tabular}


Figura 1

Índice CPOD médio por gênero nos oito municípios participantes.

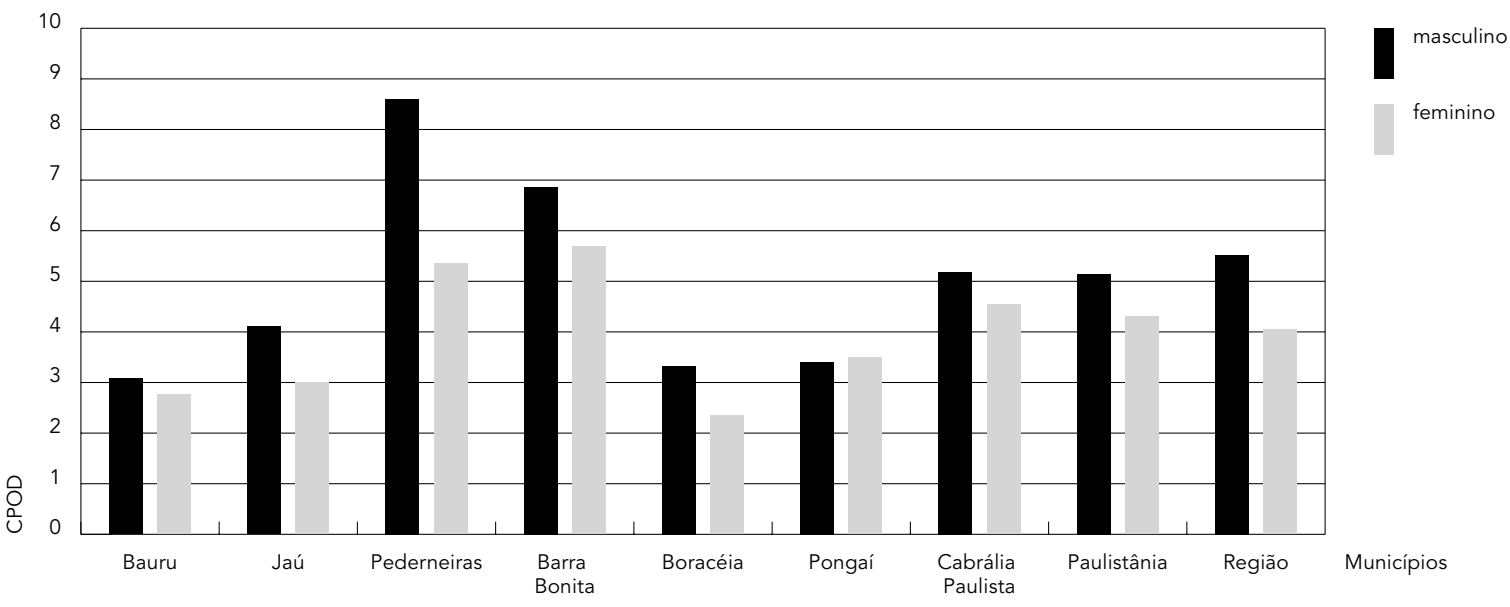

Figura 2

Percentual de escolares que apresentaram CPOD 4-18, em cada município.

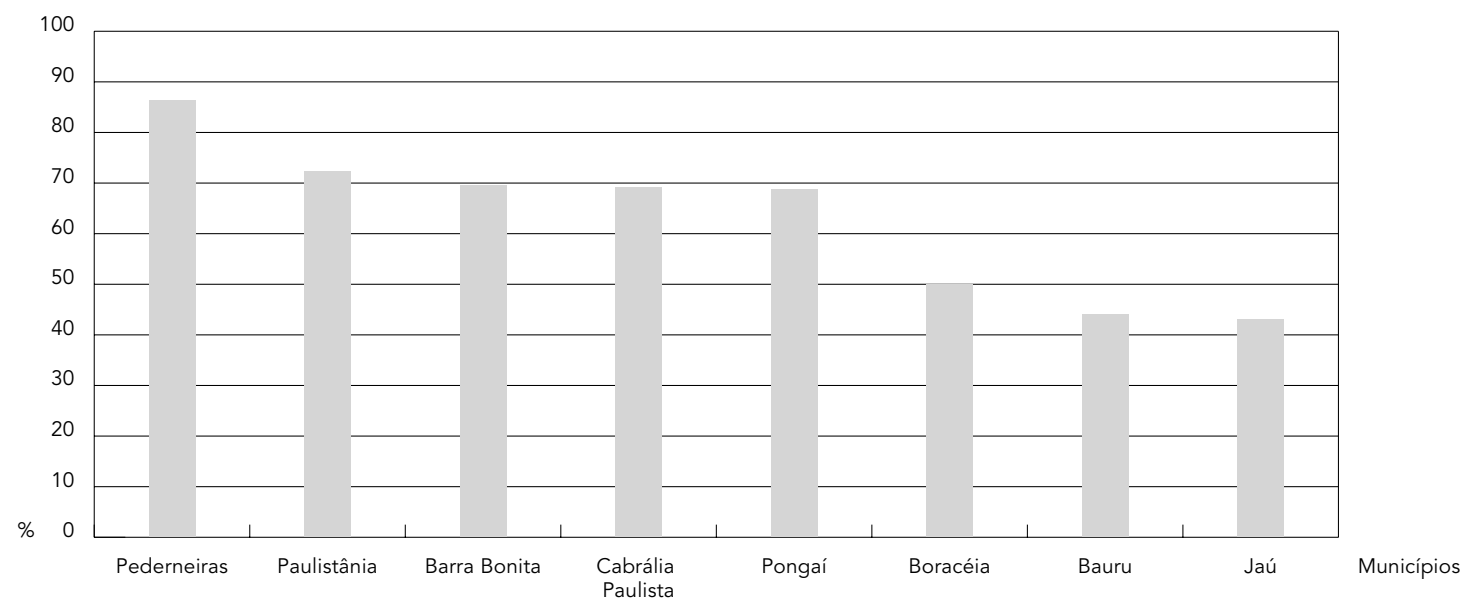

sabido a importante participação do flúor nesse processo. Pinto (2000), cita como possíveis razões para o declínio da cárie: dentifrício fluoretado, água fluoretada, bochechos com flúor, aplicações tópicas de flúor, mudança no consumo de açúcar, melhoria na limpeza dos dentes, educação em saúde bucal, mudança no diagnóstico de cárie e melhoria das condições sócio-econômicas. Pode-se acrescentar a in- gestão por diferentes fontes de flúor e o "efeito halo". Muitos produtos consumidos pela população apresentam flúor em sua composição, como os sucos em pó, leite em pó, achocolatados e ainda os produtos manufaturados em regiões fluoretadas e consumidos em regiões não fluoretadas. A interação desses fatores propiciaram a convergência entre municípios fluoretados e não fluoretados, em relação à preva- 
Percentual de crianças livres de cárie aos 12 anos de idade, em cada município da pesquisa.
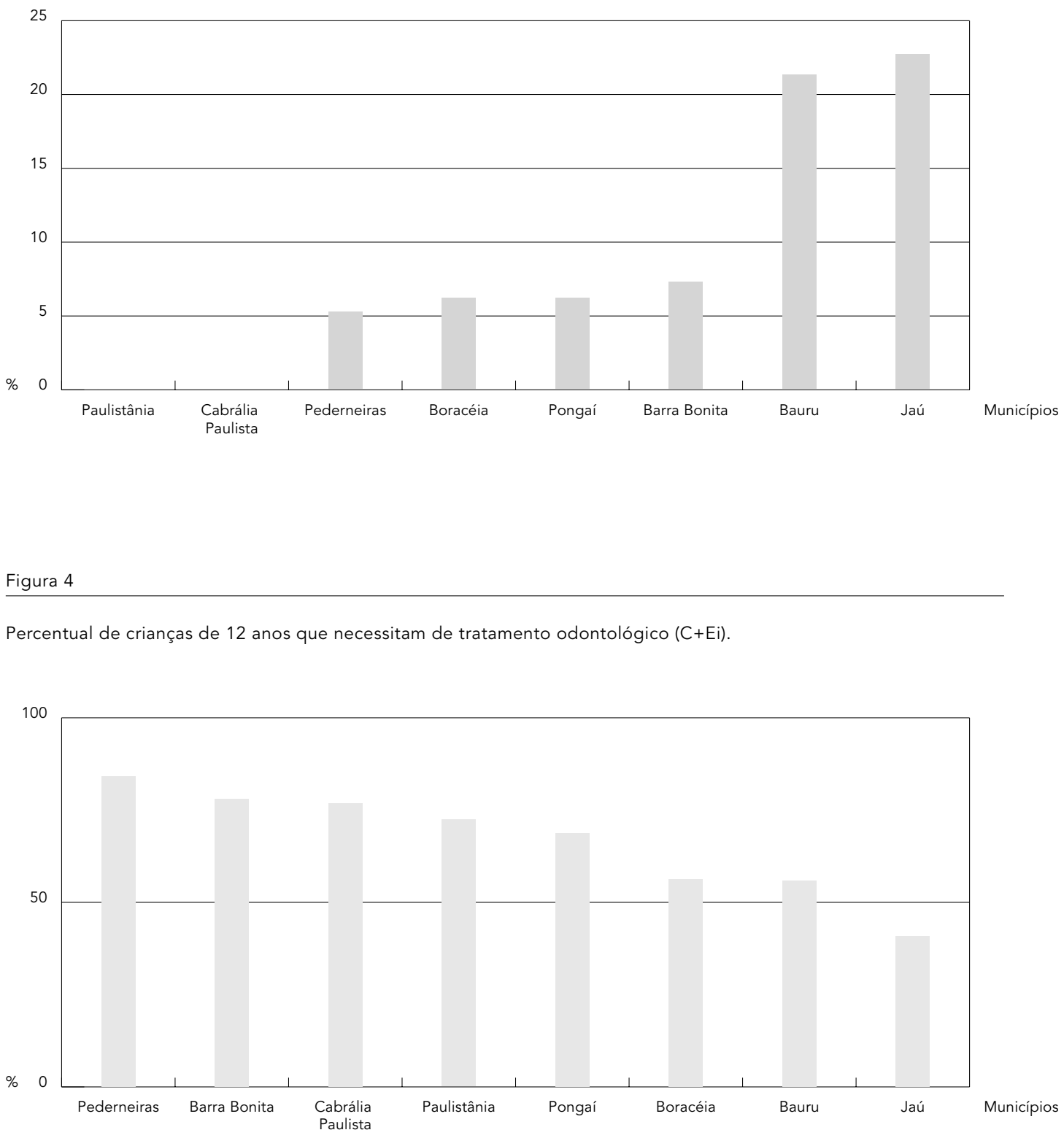

lência de cárie dentária. De Liefde (1998), na Nova Zelândia, e Angelilo et al. (1999), na Itália, observaram este fenômeno em seus estudos, correlacionaram o novo perfil epidemiológico da cárie dentária à esta grande ingestão de flúor pela população. No Brasil, pode-se destacar o estudo de Pereira et al. (2000), que avaliou o declínio de cárie dentária em cidade não fluoretada e o aumento da fluorose. Os autores ressaltaram como possíveis fatores causais para o aumento da fluorose e o declínio da cárie, o consumo de alimentos industrializados em regiões fluoretadas e o uso de dentifrícios, sugerindo o monitoramento da fluorose mesmo em regiões não fluoretadas. Este estudo demonstrou que não houve diferença estatisticamente significante ao comparar municípios de mesmo porte com ou sem flúor na água de 


\begin{tabular}{ll} 
Tabela 2 \\
\hline $\begin{array}{l}\text { Teste de Tukey para comparações individuais } \\
\text { de CPOD. }\end{array}$ \\
\begin{tabular}{lll} 
Porte do & Flúor & Média \\
município & & \\
\hline $\begin{array}{l}\text { Grande } \\
\text { Pequeno }\end{array}$ & Sim & 2,92 \\
Grande & Sim & 3,08 \\
Pequeno & Não & 3,45 \\
Médio & Não & 4,82 \\
Médio & Não & 6,47 \\
& Sim & 7,06
\end{tabular}
\end{tabular}

Grupos unidos por barra vertical não possuem diferença estatisticamente significante entre si.

abastecimento, demonstrando a presença do fenômeno da convergência nesta região. A prevalência de cárie não difere muito entre municípios fluoretados e não fluoretados, muito provavelmente devido à exposição da população aos diferentes veículos com flúor. O declínio de cárie, alcançado através do método da fluoretação de águas de abastecimento público, oferece uma redução de até $65 \%$ (Lopes \& Bastos, 1988) e o contínuo declínio parece estar condicionado ao efeito sinérgico de outros métodos preventivos. Lopes \& Bastos (1988) relacionaram muitas pesquisas onde a maior prevalência de cárie ocorreu no gênero feminino, haja vista a erupção precoce dos dentes neste gênero. Ao separar a amostra segundo o gênero (Tabela 1, Figura 1), os resultados diferenciam da maioria dos trabalhos da literatura especializada, que afirmam que a maior prevalência de cárie ocorre no gênero feminino. Os resultados da região centro-oeste do Estado de São Paulo demonstraram maior prevalência para o gênero masculino, com exceção do Município de Pongaí. Essa tendência deve ser melhor investigada para que futuras afirmações possam ser elaboradas. Moalic et al. (1999), na França, encontrou resultados semelhantes, e afirmou que estes valores são no mínimo conflitantes, mas que a população em seu estudo fora muito homogênea e a cárie foi mais prevalente nos meninos. Um estudo realizado na cidade de Bauru, por Franzolin et al. (2000), com a participação de 360 escolares aos 12 anos de idade, demonstrou que a maior prevalência ocorreu para o gênero masculino independente da cor/raça, fato este que reafirma a tendência de maior prevalência de cárie dentária no gênero masculino nessa região. Algumas justificativas podem ser elaboradas em favor do gê- nero feminino, como a maior conscientização ao auto-cuidado, realização melhor e por mais vezes ao dia do controle mecânico de placa, ou analisando por outra vertente, os meninos talvez tenham um menor rigor com a própria saúde geral. Muitas investigações são necessárias para justificar as possíveis mudanças na ocorrência da cárie dentária, doença esta já tão estudada mas que ainda reserva inovações frente à tendência ao declínio em sua prevalência e severidade. No final da década de 80 , quando foram introduzidos no mercado brasileiro os dentifrícios fluoretados, houve uma mudança no perfil epidemiológico de cárie dentária, ocorrendo declínio no índice CPOD e aumento no porcentual de indivíduos livres de cárie. A OMS (WHO, 1981) propôs metas para o ano 2000, em que crianças brasileiras aos 12 anos de idade deveriam apresentar CPOD igual ou menor que 3,0. A Figura 2 demonstra que na cidade de Pederneiras, $86,31 \%$ das crianças não atingiram esta meta, Paulistânia, Barra Bonita e Cabrália Paulista, por volta de $70 \%$ apresentaram CPOD entre 4 e 18 e os melhores resultados foram encontrados em Bauru e Jaú, com 44 e $43,18 \%$, respectivamente. O padrão de cárie pode ser investigado através do CPOD e do porcentual de livres de cárie. As cidades de Paulistânia e Cabrália Paulista (pequeno porte, sem flúor) apresentaram o percentual de livres de cárie igual a zero, situação que precisa ser modificada. Esses municípios deveriam fluoretar a água de abastecimento público, oferecendo a sua população um método preventivo de ampla cobertura e baixo custo. Nas cidades de Bauru e Jaú, mais de $20 \%$ dos escolares apresentaram-se livres de cárie na idade em estudo, mas ainda estão longe dos valores encontrados em outras cidades brasileiras. Marcenes \& Bonecker (2000) relacionaram alguns estudos de prevalência de cárie, entre eles destacase o de Araraquara em 1995 (CPOD aos 12 anos foi igual 2,60), ou de Blumenau em 1998 (CPOD foi igual a 1,39 ), que quando comparados à região centro-oeste do Estado de São Paulo, verifica-se a necessidade da implementação de um programa em saúde bucal, que seja efetivo. A utilização de métodos preventivos combinados que possam melhorar as condições de saúde bucal na região, haja vista a ação do flúor ser base fundamental na prevenção de cárie dentária. Os autores afirmam que os benefícios na redução da doença não podem ser atribuídos unicamente à fluoretação de águas de abastecimento público, porém ao efeito interativo dos diferentes métodos, como a ingestão de parte da quantidade de dentifrícios fluoretados usados nas escovações dentais, o "efeito halo" (pro- 
dutos produzidos em regiões fluoretadas e consumidos em regiões não fluoretadas), o consumo de água engarrafada e o uso de sistemas de purificação de água em residências, fatores que provavelmente interagem corroborando para o declínio da cárie. Esse declínio não está ocorrendo de forma homogênea, o que se pode observar é que um pequeno grupo representa a grande maioria dos problemas em saúde bucal, e um porcentual maior se apresenta livres de cárie ou com poucas lesões, demonstrando a ocorrência da polarização da cárie dentária. O fenômeno da polarização ou dos grupos de alta atividade de cárie dentária tem sido documentado, tanto em crianças como em adultos, em inúmeros países industrializados como a Finlândia, o Reino Unido, a Austrália e também em nações em desenvolvimento como a Arábia Saudita (Weyne, 1997). No Brasil, em 1995, na cidade de Santos, foram encontrados indicadores do declínio heterogêneo de cárie. A amostra aos 12 anos de idade apresentou-se em $45,4 \%$ livres de cárie e $20,2 \%$ com CPOD entre 4 a 15, centralizando a maior parte das necessidades de tratamento (FSP-USP, 1995).

O planejamento de políticas públicas de saúde deve estar pautado no conhecimento das necessidades da população, correlacionando causas, efeitos e soluções possíveis dos problemas, dimensionando melhor os recursos disponíveis. Acheson (1978) afirma que há duas abordagens diferentes para a definição das necessidades de cuidados de saúde. Uma visão "humanitária" que se refere a algum distúrbio na saúde e no bem-estar, e outra "realística" na qual a necessidade deve ser reconhecida apenas quando existir alguma intervenção médica, e que altere de modo favorável o prognóstico do tratamento a um custo razoável. Pinto (2000) ressalta que a importância na indicação do tratamento a ser realizado, depende em grande parte da capacidade de oferta de serviços. A região como um todo necessita de assistência odontológica, tanto de programas de promoção de saúde bucal, mas também os que ofereçam a tríade educação/prevenção/ restauração, haja vista os altos porcentuais de necessidades de tratamento (Figura 4) nos municípios da região. Segundo as respostas a um questionário encaminhado às secretarias dos municípios do estudo, todos eles oferecem programas educativos e preventivos aos escolares, e as cidades de médio e grande porte também oferecem programas aos pré-escolares, seguindo as recomendações da DIR-X. Pode-se observar que nos municípios de grande porte, Bauru e Jaú, está ocorrendo uma redução considerável no porcentual de crianças livres de cárie entre os 5 e 12 anos de idade. Aos cinco anos, aproximadamente $55 \%$ deles não tiveram experiência de cárie e aos 12 , reduz a $22 \%$. Esses resultados demonstram que há necessidade da implementação de programas específicos nesta faixa etária para que possam atingir os 12 anos sem lesões de cárie. Entre os municípios do estudo, em Pederneiras os escolares apresentaram a maior necessidade de tratamento, quer no volume ou na gravidade, necessitando de maior número de procedimentos por criança. Sugere-se que um Índice de Severidade da Lesão de cárie dentária (ISL) possa ser elaborado para dimensionar os níveis aceitáveis de cárie dentária para população brasileira. O CPOD médio aos 12 anos, na região centro-oeste, foi 4,82 , não atingindo as metas da OMS para o ano 2000. A meta para o ano de 2010, de CPOD menor que 1,0, está distante da realidade da região. Neste início de milênio, frente às inovadoras tecnologias, buscando atingir uma melhor qualidade de vida, a saúde bucal está em alerta e é preocupante na região centro-oeste do Estado de São Paulo.

\section{Referências}

ACHESON, R. M., 1978. The definition and identification of need for health care. Journal of Epidemiology and Community Health, 32:10-15.

ANGELILLO, I. F.; TORRE, I.; NOBILE, C. G. \& VILLARI, P., 1999. Caries fluorosis prevalence in communities with different concentrations of fluoride in the water. Caries Research, 33:114-122.

CLARK, D. C. \& BERKOWITZ, J., 1997. The influence of various fluoride exposures on the prevalence of esthetic problems resulting from dental fluorosis. Journal of Public Health Dentistry, 57:144149. 
DE LIEFDE, B., 1998. The decline of caries in New Zealand over the past 40 years. New Zealand Dental Journal, 94:109-113.

DINI, E. L.; HOLT, R. D. \& BEDI, R., 1998. Comparison of two indices of caries patterns in 3-6 year old Brazilian children from area with different fluoridation histories. International Dental Journal, 48:378-385.

FRANZOLIN, S. O. B.; GONÇALVES, A. \& PADOVANI, C. R., 2000. Prevalência fluorose e cárie dentária em escolares de Bauru - São Paulo, com diferentes serviços de abastecimento de água. In: 1a Jornada Científica do Uso Racional do Flúor, Anais, p. 6. Bauru: Faculdade de Odontologia de Bauru, Universidade de São Paulo.

FSP-USP (Faculdade de Saúde Pública, Universidade de São Paulo), 1995. Levantamento Epidemiológico em Saúde Bucal: Santos, SP, 1995. Relatório. São Paulo: FSP-USP.

FSP-USP (Faculdade de Saúde Pública, Universidade de São Paulo), 1999. Levantamento Epidemiológico de Saúde Bucal - Estado de São Paulo, 1998. São Paulo: FSP-USP.

LOPES, E. S. \& BASTOS, J. R. M.,1988. Odontologia Preventiva e Social. Bauru: Faculdade de Odontologia de Bauru.

MARCENES, W. \& BONECKER, J. S. M., 2000. Aspectos epidemiológicos e sociais das doenças bucais. In: Promoção de Saúde Bucal na Clínica Odontológica (Y. P. Buischi, org.), pp. 73-98, São Paulo: Artes Médicas.

MOALIC, E.; ZERILLI, A.; CAPO-CHICHI, S. \& APOVI, G., 1999. Oral and dental health of population of school children from the Zou region of Benin. Santé, 9:273-276.

MORGAN, L.; ALLRED, E.; TAVARES, M.; BELLINGER, D. \& NEEDLEMAN, H., 1998. Investigation of the possible associations between fluorosis, fluoride exposure and chilhood behavior problems. Pediatric Dentistry, 20:244-252.
MS (Ministério da Saúde), 1988. Levantamento Epidemiológico em Saúde Bucal. Brasil, Zona Urbana, 1986. Brasília: MS.

MS (Ministério da Saúde), 1996. Levantamento Epidemiológico em Saúde Bucal nas Capitais Brasileiras, em 1996. Brasília: MS.

MURRAY, J. J., 1994. Comments on the Conference (Second International Conference on Declining Caries). International Dental Journal, 44(Sup. 1): 457-458.

NARVAI, P. C.; FRAZÃO, P. \& CASTELLANOS, R. A., 1999. Declínio na experiência de cárie em dentes permanentes de escolares brasileiros no final do século XX. Revista Odontologia e Sociedade, 1:2529.

PEREIRA, A. C.; DA CUNHA, F. L.; MENEGHIM, M. C. \& WERNER, C. W., 2000. Dental caries and fluorosis prevalence study in a nonfluoridated Brazilian community: Trend analysis and toothpaste association. Journal of Dentistry for Children, 6:132135.

PINTO, V. G., 1997. Epidemiologia da doenças bucais no Brasil. In: Promoção de Saúde Bucal (Associação Brasileira de Odontologia de Promoção de Saúde, org.), pp. 27-41, São Paulo: Artes Médicas.

PINTO, V. G., 2000. Saúde Bucal Coletiva. 4a Ed. São Paulo: Editora Santos.

SILVA, B. B. \& MALTZ, M., 2001. Prevalência de cárie e fluorose em escolares de 12 anos de Porto AlegreRS, Brasil, 1998/1999. Pesquisa Odontológica Brasileira, 15:208-214.

WEYNE, S. C. A., 1997. A construção do paradigma de promoção de saúde - Um novo desafio para as novas gerações. In: Promoção de Saúde Bucal (Associação Brasileira de Odontologia de Promoção de Saúde, org.), pp. 1-26, São Paulo: Artes Médicas.

WHO (World Health Organization), 1981. Global Strategy for Health for All by Year 2000. Health for All Series 3. Geneva: WHO.

Recebido em 26 de julho de 2001

Versão final reapresentada em 23 de dezembro de 2001 Aprovado em 4 de março de 2002 